\title{
Successful endoscopic management of 3 cases of translocated intrauterine devices: a case report
}

\author{
Xiaoyan Han, Hua Yang \\ Department of Gynecology and Obstetrics, Beijing Friendship Hospital, Capital Medical University, Beijing, China \\ Correspondence to: Hua Yang. Department of Gynecology and Obstetrics, Beijing Friendship Hospital, Capital Medical University, Beijing 100050, \\ China. Email: yanghuawst@163.com.
}

\begin{abstract}
Intrauterine devices (IUDs) are the most popular form of contraception globally; however, the use of IUDs carries some risks. IUD migration is a rare but serious complication that occurs after insertion, sometimes years later. Herein, we report 3 cases of translocated IUDs removed by endoscopy and describe the clinical characteristics and intraoperative findings of these patients. Case 1 was a 46-year-old woman who presented with upper abdominal pain. Gastroscopy showed the presence of an IUD inside the stomach. Digestive endoscopy performed at our hospital showed a T-shaped foreign body embedded in the gastric wall at the junction between the gastric antrum and the gastric body. The IUD was removed endoscopically without complications. Case 2 was a 36-year-old woman who had twice undergone cesarean section. Two months before presenting at our hospital, the patient experienced external hemorrhoid. During anal suppository treatment, she felt a lump inside the rectum. Pelvic computed tomography revealed a small, circular dense shadow with an approximate diameter of $0.4 \mathrm{~cm}$ in the upper intestinal cavity of the rectum. Subsequently, the patient received transanal endoscopy and rectal foreign-body removal surgery. Case 3 was a 40-year-old woman. She had failed to remove an IUD in other 2 hospitals. On the basis of pelvic computed tomography, the IUD was suspected to have perforated the bladder. Cystoscopy showed that part of the IUD was surrounded by stones. Laser lithotripsy was performed to expose the IUD completely. Under hysteroscopy, the IUD could be seen in the middle of the uterine cavity; 1 end was located on the right uterine horn, and the other was protruding out of the uterus. During the operation, the IUD was broken into two parts and one part was removed through the vagina. The cystoscope was placed again, and the remaining part of the IUD was smoothly removed. Although IUD migration has a low incidence, it can have a huge impact on patients' physical and emotional health. Migrated IUDs must be removed immediately. Hysteroscopy and digestive endoscopy are effective approaches for removing migrated IUDs.
\end{abstract}

Keywords: Intrauterine device (IUD); migration; hysteroscopy; digestive endoscopy; case report

Submitted Dec 10, 2020. Accepted for publication Feb 04, 2021.

doi: 10.21037/apm-21-184

View this article at: http://dx.doi.org/10.21037/apm-21-184

\section{Introduction}

Intrauterine devices (IUDs) are a common female contraceptive method in China. IUDs are widely used due to their high effectiveness in preventing pregnancy. Although IUDs have proven to be a safe form of contraception for many women, as with any birth control methods, potential side effects and complications exist. Some common complications include abdominal pain and abnormal bleeding; less common complications include pelvic inflammatory disease, IUD expulsion, IUD retraction into the cervix or uterus, and uterine perforation. The occurrence of these complications appears to be associated with the type of device, time of insertion, skill of the operator, position of the uterus, and degree of follow-up. The occurrence of translocated IUD is not common, and mainly affects the bladder, and pelvic and 


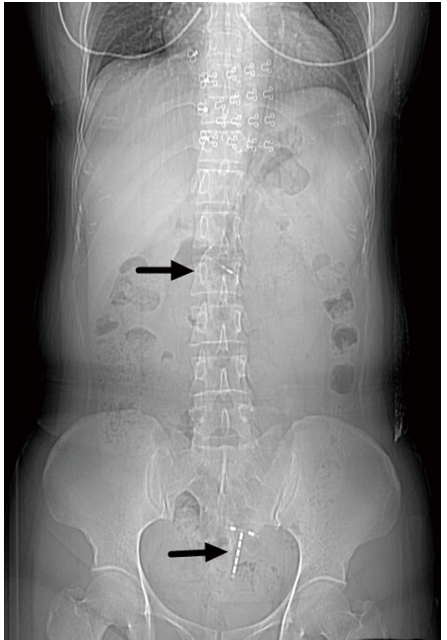

Figure 1 Computed tomography image showed the echo of an intrauterine device in the upper abdominal cavity (indicated by the top black arrow) and that of another intrauterine device in the pelvic cavity (indicated by the bottom black arrow).

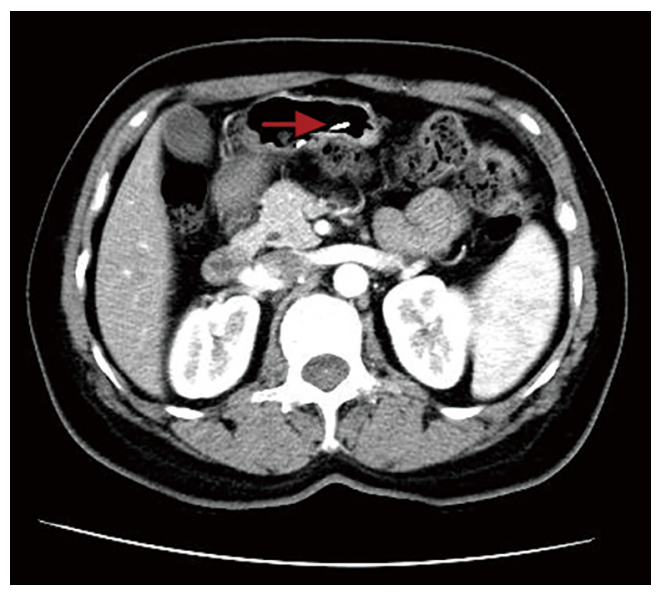

Figure 2 Abdominal contrast-enhanced computed tomography showed the high-density metal shadow (indicated by the red arrow) in the lower part of the stomach.

abdominal cavities. The intestinal tract is the organ most often affected. IUD migration can cause different clinical manifestations (1,2). Many patients with translocated IUD are asymptomatic and present with pregnancy or missing IUD strings. A small number of patients present with acute symptoms of bowel obstruction or perforation. In the past, ectopic IUDs in asymptomatic patients were not removed; however, today, most experts advise removal of any perforated IUD. Here, we report 3 cases in which translocated IUDs were successfully removed by endoscopy, two of which migrated to the bladder and rectum, the other one migrated to the gastric cavity which was extremely rare in the literature.

We present the following article in accordance with the CARE reporting checklist (available at http://dx.doi. org/10.21037/apm-21-184).

\section{Case presentation}

Case 1

Case 1 was a 46-year-old patient. Her gravidity and parity were 5 and 2, respectively. She had a spontaneous vaginal delivery in 1990. In 1991, a Copper-T IUD was placed. In 2003, the IUD was removed in a local hospital after the patient experienced abdominal pain, pelvic inflammatory disease, and suspected IUD migration, although the exact diagnosis was unclear. The patient's symptoms improved after postoperative anti-inflammatory therapy. In 2005, the patient delivered a full-term infant via cesarean section. In the years that followed, she had 2 first-trimester abortions. In 2009, the patient underwent a right salpingectomy in a local hospital due to ectopic pregnancy in the right fallopian tube. In 2013, a second IUD (another Copper-T IUD) was inserted.

The patient presented with upper abdominal pain with no obvious cause, which showed no improvement after treatment with oral painkillers. Gastroscopy performed in another hospital showed the presence of an IUD inside the stomach. The patient repeatedly denied that she had swallowed an IUD. She was subsequently referred to our center for further investigation. Computed tomography image showing the echo of an intrauterine device in the upper abdominal cavity and that of another intrauterine device in the pelvic cavity. Abdominal contrast-enhanced computed tomography showing the high-density metal shadow in the lower part of the stomach (Figures 1,2). Digestive endoscopy performed at our hospital on January 14, 2016 revealed a T-shaped foreign body at the junction between the gastric antrum and the gastric body (near the posterior wall); the foreign body was twisted with silklike material, and 1 end was embedded in the gastric wall (Figure 3). Gynecological ultrasound indicated normal positioning of the IUD in the uterus. A T-shaped IUD was removed endoscopically without complications (Figures 4,5). The patient received postoperative anti-inflammatory treatment, and was discharged after her condition had improved. During the 2-month outpatient follow-up, the 


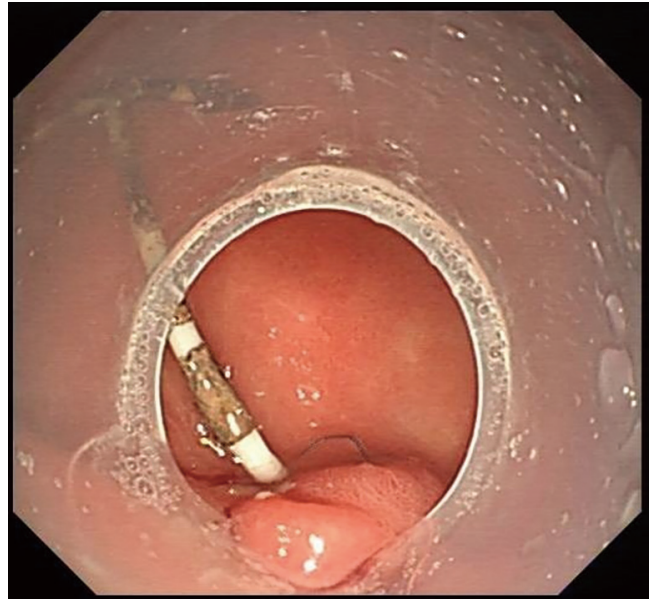

Figure 3 Gastroscopy performed in our hospital revealed a T-shaped foreign body at the junction of the gastric antrum and the gastric body (near the posterior wall).

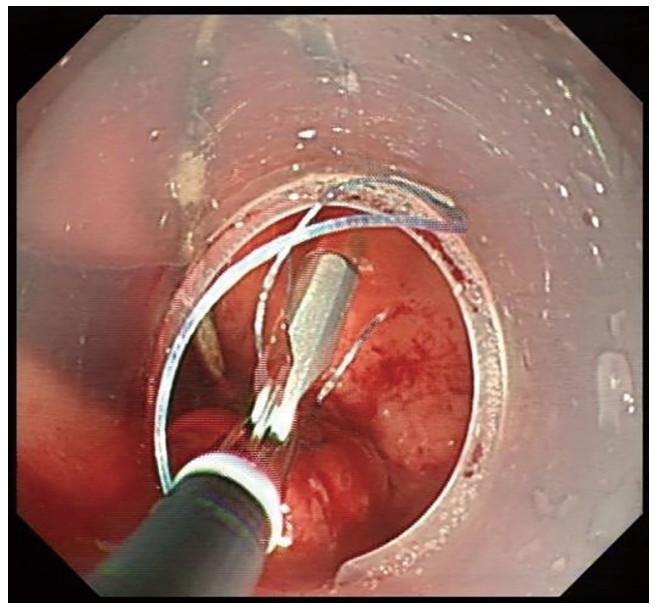

Figure 4 A migrated intrauterine device was removed by gastroscopy without complications. The tail fiber of the intrauterine device was located inside the muscular layer of the gastric wall.

patient reported no gastric discomfort and experienced regular menstrual cycles, and the positioning of the IUD in uterus was normal.

\section{Case 2}

Case 2 was a 36-year-old patient, gravidity 2 parity 2, who had twice successfully undergone cesarean section (in 2002 and 2007). In 2004, the patient received IUD insertion

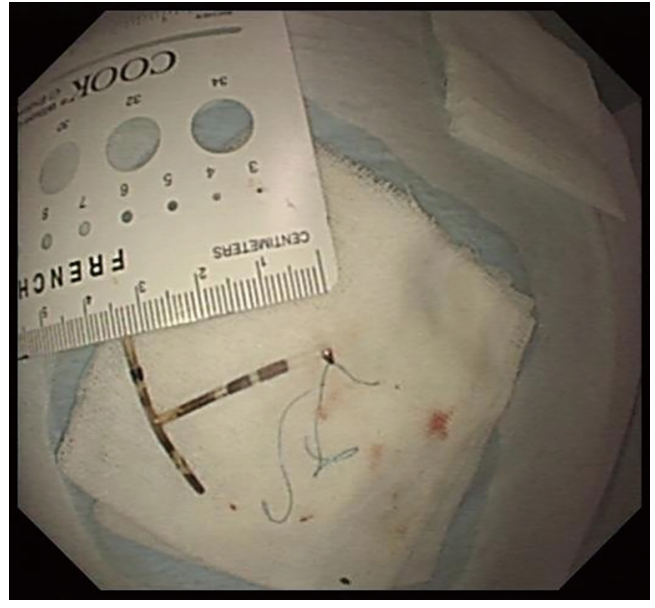

Figure 5 The migrated device was a metal T-shaped intrauterine device with tail fibers.

in another hospital. In 2005, the patient underwent early abortion in other hospital. The IUD was not found inside the uterine cavity during the operation; thus, it was considered to have self-discharged. In 2007, the patient delivered a second child by cesarean section. After surgery, she was again implanted with an IUD. The patient did not experience any discomfort after IUD insertion, and she did not attend regular follow-up. One year before presenting at our hospital, the patient started to have frequent defecation (brown thin stools, 2 to 3 times a day) with no obvious cause. The patient had no abdominal pain, abdominal distension, chills, fever, nausea, vomiting, tenesmus, or sagging sensation in the anus, and she did not visit a hospital. Two months before admitting to our hospital, the patient's external hemorrhoids relapsed. When inserting anal suppositories, she noticed a lump inside the rectum; she subsequently visited the hospital.

Gynecological ultrasound examination showed normal positioning of the IUD inside the uterine cavity. The patient underwent surgery in another hospital to remove the IUD from the uterus. After surgery, the patient visited our hospital's surgical clinic. Pelvic computed tomography (CT) showed a small, circular, dense shadow in the rectum and mixed-density content in the sigmoid colon (Figure 6). Anal examination showed external hemorrhoids at the anus. At the 7 o'clock position, a hard, thin, stick-like, metal foreign body could be felt at a $7-\mathrm{cm}$ depth from the entrance to the anus, while the residual anorectal mucosa was smooth. Gynecological ultrasound showed no abnormal lesion in the pelvic cavity. Pelvic X-ray displayed a small, circular, dense 


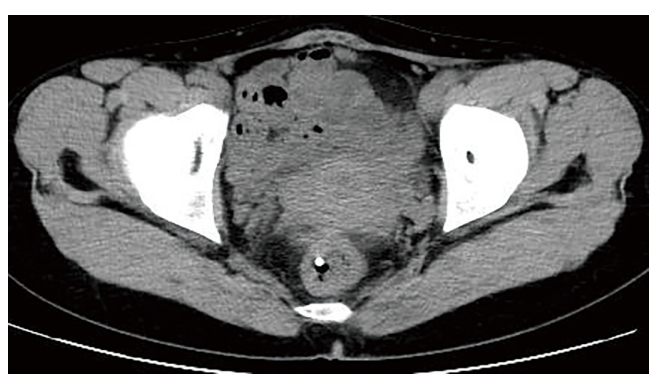

Figure 6 Pelvic computed tomography revealed a small, circular, dense shadow approximately $0.4 \mathrm{~cm}$ in diameter in the upper rectal cavity.

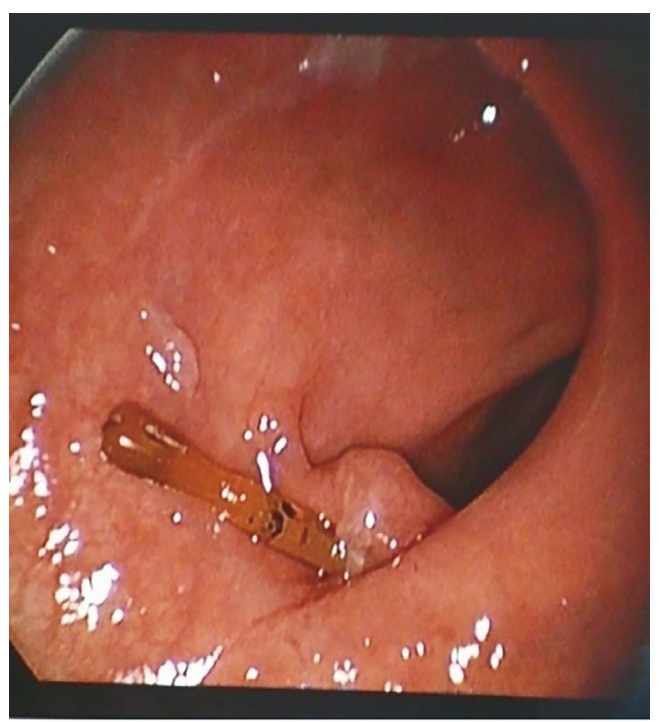

Figure 7 Transanal endoscopy image showed the IUD incarcerated in the anterior rectal wall; the top and bottom parts of the IUD were exposed, and the remaining part was embedded in the mucosa.

shadow, which was possibly a pelvic foreign body. Pelvic CT revealed a small, circular, dense shadow approximately $0.4 \mathrm{~cm}$ in diameter in the upper rectal cavity. After adequate preoperative preparation, the patient underwent transanal endoscopy and surgery to remove the rectal foreign body in August 28, 2017. During the operation, an IUD could be seen to be incarcerated in the anterior rectal wall; the top and bottom parts of the IUD were exposed, and the remaining part was embedded in the mucosa (Figure 7). An ultrasonic knife was used to incise part of the rectal mucosa layer, blunt dissection was performed, and the IUD was completely removed. Further exploration showed that the muscular layer of the rectum was complete. The operation went smoothly, and intraoperative blood loss was about $5 \mathrm{~mL}$. Postoperative evaluation by pelvic CT showed no trace of the small, circular, dense shadow in the rectum. The patient was discharged from hospital 2 days later, after which, she was regularly followed up and experienced no discomfort.

\section{Case 3}

Case 3 was a 40-year-old patient who was admitted to the hospital on December 10, 2019, with an IUD implanted for more than 3 years. The patient had failed to remove the IUD in other 2 hospitals. The patient received the IUD insertion in May 2016. She underwent IUD removal surgery at a local health center on July 152019 due to the downward of the IUD. During the operation, a ring could be felt at the inner mouth of the cervix and proved difficult to remove. IUD incarceration was suspected, and the operation was stopped. On November 12 2019, the patient underwent hysteroscopy to remove the IUD in another hospital. During the operation, the deep incarceration of IUD was observed. Consequently, the operation was stopped, and the patient was transferred to our hospital.

On November 20, 2019, the patient underwent pelvic CT in our hospital, which suggested that the IUD had perforated into the bladder. Vaginal ultrasound showed a strong echo of the IUD in the lower segment of the uterine cavity and the cervical canal. Part of the IUD was penetrating the anterior lip of the cervix and was perforating into the bladder (Figure 8). Computed tomography image showed he IUD had perforated into the bladder (Figure 9). After preoperative preparation, the patient received cystoscopy under intravenous anesthesia. Cystoscopy showed that part of the IUD was surrounded by stones (Figure 10). Laser lithotripsy was performed to expose the IUD ring completely. Under hysteroscopy, the IUD could be seen in the middle of the uterine cavity; 1 end was positioned on the right uterine horn, and the other was sticking out of the uterus. The IUD was broken into two parts during the operation, and one part of it was removed through the vagina. The cystoscope was placed again, and the remaining part of the IUD was removed smoothly (Figure 11). Finally, hysteroscopy was performed again, and no residual foreign body was observed. The patient was discharged from the hospital with a urinary catheter 2 days after surgery. After 3 weeks, the urinary catheter was removed in the urology clinic. The patient was able to 


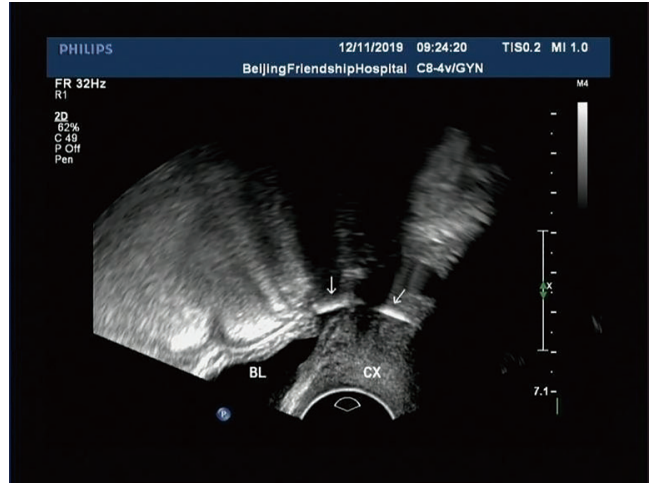

Figure 8 Vaginal ultrasound showed a strong echo of the IUD in the lower segment of the uterine cavity and the cervical canal. Part of the IUD was penetrating the anterior lip of the cervix and was perforating into the bladder.

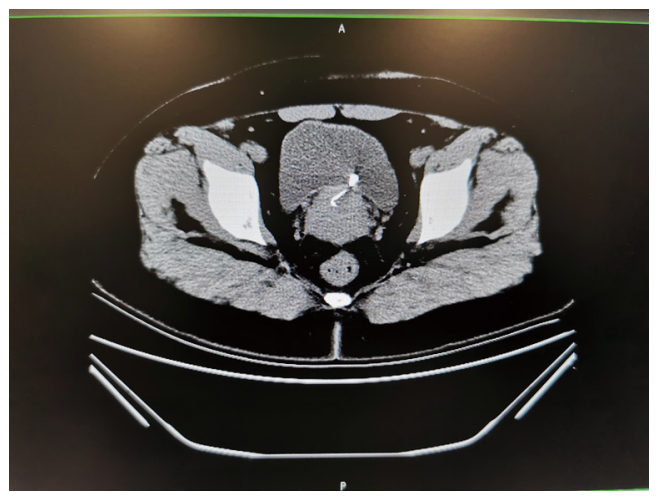

Figure 9 Computed tomography image showed he IUD had perforated into the bladder.

urinate smoothly with no discomfort, and no abnormalities were detected during gynecological ultrasound. All procedures performed in studies involving human participants were in accordance with the ethical standards of the institutional and/or national research committee(s) and with the Helsinki Declaration (as revised in 2013). Written informed consent was obtained from the patient.

\section{Discussion}

An IUD is a long-acting, economic, and reversible female contraceptive method that is commonly used in China. The common side effects and complications of IUD placement include abnormal uterine bleeding, pain, IUD incarceration, perforation beyond the uterus used by IUD migration, and

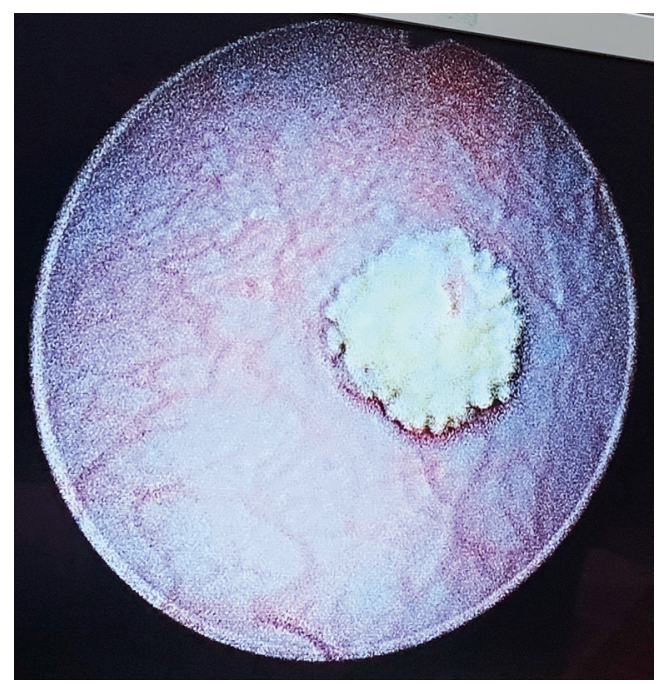

Figure 10 Cystoscopy image showed part of the IUD was surrounded by stones.

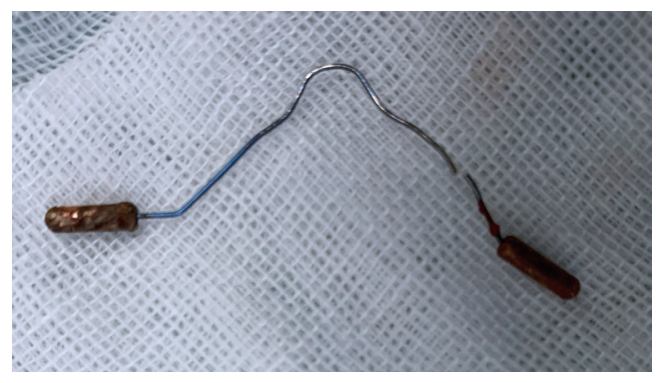

Figure 11 The ectopic intrauterine device with fracture during the removal process.

unplanned pregnancy. Among these complications, IUD migration is particularly severe, as it can cause varying degrees of abdominal pain, pelvic infection, intestinal adhesions, bowel obstruction, urinary tract infection, and even organ dysfunction, and it often requires surgical treatment (1). IUD migration can be classified into 3 types: (I) partial migration, in which part of the IUD becomes embedded in the myometrium; (II) complete migration, in which the IUD becomes completely embedded in the myometrium; and (III) migration outside the uterus, in which the IUD leaves the uterine cavity entirely and enters the pelvic cavity or other areas of the abdominal cavity $(1,2)$. Migration of an IUD outside the uterus is rare, and mainly affects the bladder, and pelvic and abdominal cavities (3-7). The IUDs in the 3 patients described herein were ectopic 
to the stomach, rectum, and bladder, respectively, and all were extrauterine ectopic cases.

Most patients with ectopic IUD exhibit no obvious symptoms, and most cases are identified after accidental pregnancy or during routine examination $(8,9)$. When an IUD is ectopic, about $15 \%$ of the perforated IUD will become embedded in or invade the surrounding organs. The intestinal tract is the organ most often affected. IUD ectopic to intestinal tract can manifest as intestinal perforation, intestinal obstruction, mesenteric penetration, intestinal infarction, rectal stenosis, or uterine rectal fistula, and patients develop symptoms such as abdominal pain, diarrhea, or fever $(2,8)$. The clinical manifestations of the 2 patients in our study included epigastric pain, stool changes, and difficulty defecating. The patient with an IUD ectopic to the bladder exhibited no clinical symptoms.

IUD migration can be diagnosed using techniques including radiography, ultrasound, and CT. X-rays cannot penetrate through metal-bearing IUDs, and thus can be used to determine the presence of an IUD inside the body. Meanwhile, ultrasound examination can help physicians to determine the site, depth, and scope of IUD incarceration, and can also be used to determine whether the IUD has become fractured or deformed; thus, it is the preferred modality for evaluating IUD migration, especially given its safety, accuracy, and reliability. CT can also be used to locate a migrated IUD, and it can clearly reveal the relationships of the IUD with the uterus and its adjacent organs $(8,9)$. All 3 of our patients underwent CT examination; their IUDs were found in the stomach, rectum, and bladder, and were finally diagnosed by gastroscopy, colonoscopy, and cystoscopy, respectively.

The potential causes of IUD migration include the medical staff performing the IUD insertion having limited skills or experience, inappropriate device or model selection, inappropriate timing of IUD insertion, and the physical condition of the patient (2). Risk factors for IUD migration include: (I) a poorly positioned uterus and rough technique during IUD placement; (II) a soft uterine wall that is easily penetrated (for instance, due to breastfeeding or after an abortion); (III) direct injury during IUD insertion and the chronic erosive effect of the IUD on the uterine wall; (IV) uterine scarring; and (V) postmenopausal atrophy of the uterus and shrinkage of the uterine cavity, which can easily cause the IUD to become embedded in the muscular layer and results in difficulty in IUD withdrawal. Thus, patients with an IUD should have it removed within 1 year after menopause. IUD migration may also be associated with the natural contractions of the uterus, bladder contraction, visceral motility, and peritoneal fluid flow $(3,8)$.

We speculate that the causes of ectopic IUD in our 3 patients may be as follows: (I) the incision ring moved along the uterine wall, leaving the uterus and entering the abdominal cavity; (II) under the action of visceral motility and inflammation, the intrauterine ring moved to the gastric serosa, rectum serosa, and bladder; the IUDs then became further penetrated into the stomach wall, intestinal wall, and bladder, and were thereby ectopic to the gastric cavity, rectal cavity, and bladder. This suggests that clinically, the position of the IUD needs to be examined regularly. Specifically, in cases of "disappeared" IUDs, the patients must be thoroughly checked, and should not simply be thought as the IUD self-discharging.

Once confirmed, the migrated IUD should be removed as soon as possible. Corresponding examinations such as ultrasound and CT should be performed before surgery to determine the specific location of the migrated IUD. IUDs that are not deeply incarcerated inside the uterine cavity can be retrieved using a vaginal hook; however, if the removal using a vaginal hook fails, or if the IUD is broken, hysteroscopy may be performed for examination and IUD removal instead (8). In addition, doctors with experience in hysteroscopic surgery should be invited to perform the operation to prevent the occurrence of secondary injuries. If the IUD has migrated to an unidentified location outside the uterine cavity, it may be removed using laparoscopy $(2,9)$. Laparoscopy provides a clear visual field and enables observation of the whole pelvic cavity; furthermore, the angle of view can be changed, which allows the operator to explore the abdominal cavity to find the migrated IUD. Laparoscopic surgery is minimally invasive and allows fast recovery; thus, it has become the preferred treatment for IUDs that have migrated outside the uterus. If an IUD cannot be located, a comprehensive examination is required to find the IUD and confirm the possible IUD expulsion (10-12). According to the specific organs of the IUD ectopic, general surgery or urological surgeons can be invited to assist in the operation. In the 3 patients in our study, the IUDs were ectopic to the stomach, rectum, and bladder; after diagnosis by gastroscopy, colonoscopy, and cystoscopy, respectively, the IUDs were successfully removed under endoscopy. Among the cases reported here, there was difficulty in removing the ring from the patient with the IUD ectopic to the bladder due to the formation of stones on the surface of the IUD. After laser lithotripsy, the IUD was successfully removed under cystoscope combined 
with hysteroscopy.

Some strategies may be useful for preventing IUD migration. For instance, before IUD insertion, gynecological examination should be performed to determine the location and size of the uterus. If the uterus is excessively anteverted or retroverted, perforation of the isthmus of the uterus can easily occur during the operation. The uterus should be returned to its normal position before the procedure to insert the IUD, and an appropriate IUD should be selected based on the depth of the uterine cavity. An excessively anteverted uterine can be corrected by pulling the posterior lip outward using cervical forceps; then, the clinician can deliver the IUD toward the uterine cavity using their right hand, while correcting and fixing the uterine position using their left hand. Particular care is needed when IUD placement is performed during lactation (10). Furthermore, an excessively large IUD can compress the uterus, gradually become embedded into the muscular layer, and migrate to outside the uterus. Therefore, it is important to select the appropriate IUD model for each patient. In addition, the IUD must be withdrawn within 1 year after menopause.

\section{Conclusions}

In summary, although IUD migration has a low incidence, it can have a huge impact on patients, both physically and emotionally. Efforts should be made to improve patients' awareness of contraception and reproductive health. In particular, women should be advised to undergo regular examinations after IUD insertion, to identify any IUDrelated problems. Migrated IUDs must be removed immediately.

\section{Acknowledgments}

Funding: None.

\section{Footnote}

Reporting Checklist: The authors have completed the CARE reporting checklist. Available at http://dx.doi.org/10.21037/ apm-21-184

Conflicts of Interest: Both authors have completed the ICMJE uniform disclosure form (available at http://dx.doi. org/10.21037/apm-21-184). The authors have no conflicts of interest to declare.
Ethical Statement: The authors are accountable for all aspects of the work in ensuring that questions related to the accuracy or integrity of any part of the work are appropriately investigated and resolved. All procedures performed in studies involving human participants were in accordance with the ethical standards of the institutional and/or national research committee(s) and with the Helsinki Declaration (as revised in 2013). Written informed consent was obtained from the patient.

Open Access Statement: This is an Open Access article distributed in accordance with the Creative Commons Attribution-NonCommercial-NoDerivs 4.0 International License (CC BY-NC-ND 4.0), which permits the noncommercial replication and distribution of the article with the strict proviso that no changes or edits are made and the original work is properly cited (including links to both the formal publication through the relevant DOI and the license). See: https://creativecommons.org/licenses/by-nc-nd/4.0/.

\section{References}

1. Şanlıkan F, Arslan O, Avcı ME, et al. Laparoscopic removal of an intrauterine device from the sigmoid colon. Pak J Med Sci 2015;31:214-6.

2. Goldstuck ND, Wildemeersch D. Role of uterine forces in intrauterine device embedment, perforation, and expulsion. Int J Womens Health 2014;6:735-44.

3. Mosley FR, Shahi N, Kurer MA. Elective surgical removal of migrated intrauterine contraceptive devices from within the peritoneal cavity: a comparison between open and laparoscopic removal. JSLS 2012;16:236-41.

4. Nowitzki KM, Hoimes ML, Chen B, et al. Ultrasonography of intrauterine devices. Ultrasonography 2015;34:183-94.

5. Tavecchia M, Burgos García A, de María Pallares P. Colorectal penetration by two intrauterine devices. Rev Esp Enferm Dig 2019;111:320-1.

6. Voulgaris AM, Lee T, Habib A, et al. The case of the migrating IUD. Practitioner 2015;259:25-7, 3.

7. Zolnierczyk P, Cendrowski K, Sawicki W. Intrauterine contraceptive device embedded in the omentum - case report. Int J Womens Health 2015;7:945-8.

8. Neumann DA, Graversen JA, Pugh SK. Intrauterine device embedded in omentum of postpartum patient with a markedly retroverted uterus: a case report. J Med Case Rep 2017;11:299. 
9. Mishra S. Translocation of Postplacental Intrauterine Device: A Rare Complication. J Obstet Gynaecol India 2016;66:707-9.

10. Rahnemai-Azar AA, Apfel T, Naghshizadian R, et al. Laparoscopic removal of migrated intrauterine device embedded in intestine. JSLS 2014;18:e2014.00122.

11. Almarhabi Y. Asymptomatic cecal perforation and ileocecal fistula after intrauterine device migration: a case report. J

Cite this article as: Han X, Yang H. Successful endoscopic management of 3 cases of translocated intrauterine devices: a case report. Ann Palliat Med 2021;10(2):2371-2378. doi: 10.21037/ apm-21-184
Surg Case Rep 2020;2020:rjaa015.

12. Sun X, Xue M, Deng X, et al. Clinical characteristic and intraoperative findings of uterine perforation patients in using of intrauterine devices (IUDs). Gynecol Surg 2018;15:3.

(English Language Editor: J. Reynolds) 